\title{
Optimization Over State Feedback Policies for Robust Control with Constraints ${ }^{1}$
}

\author{
Paul J. Goulart ${ }^{\text {a,2 }}$, Eric C. Kerrigan ${ }^{\text {b }}$, Jan M. Maciejowski ${ }^{\text {a }}$ \\ ${ }^{a}$ Department of Engineering, University of Cambridge, Trumpington Street, Cambridge CB2 1PZ, UK \\ ${ }^{\mathrm{b}}$ Department of Electrical and Electronic Engineering, Imperial College London, Exhibition Road, London SW7 2AZ, UK
}

\begin{abstract}
This paper is concerned with the optimal control of linear discrete-time systems subject to unknown but bounded state disturbances and mixed polytopic constraints on the state and input. It is shown that the class of admissible affine state feedback control policies with knowledge of prior states is equivalent to the class of admissible feedback policies that are affine functions of the past disturbance sequence. This implies that a broad class of constrained finite horizon robust and optimal control problems, where the optimization is over affine state feedback policies, can be solved in a computationally efficient fashion using convex optimization methods. This equivalence result is used to design a robust receding horizon control (RHC) state feedback policy such that the closed-loop system is input-to-state stable (ISS) and the constraints are satisfied for all time and all allowable disturbance sequences. The cost to be minimized in the associated finite horizon optimal control problem is quadratic in the disturbance-free state and input sequences. The value of the receding horizon control law can be calculated at each sample instant using a single, tractable and convex quadratic program (QP) if the disturbance set is polytopic, or a tractable second-order cone program (SOCP) if the disturbance set is given by a 2-norm bound.
\end{abstract}

Key words: Robust Control, Constraint Satisfaction, Robust Optimization, Predictive Control, Optimal Control

\section{Introduction}

This paper is concerned with the control of constrained discrete-time linear systems that are subject to additive, but bounded disturbances on the state. The main aim is to provide results that allow for the efficient computation of an optimal and stabilizing state feedback control policy that ensures a given set of state and input constraints are satisfied for all time, despite the presence of the disturbances. This is a problem that has been studied for some time now in the optimal control literature [4] and a number of different solutions are available, most of which draw on results from set invariance theory [5], $\ell_{1}$ optimal control [7] or predictive control $[22,24]$.

It is generally accepted that if disturbances are to be accounted for in the formulation of a constrained optimal control problem, then the optimization has to

Email addresses: pgoulart@alum.mit.edu (Paul J. Goulart), erickerrigan@ieee.org (Eric C. Kerrigan), jmm@eng.cam.ac.uk (Jan M. Maciejowski).

1 This paper was not presented at any IFAC meeting.

2 Corresponding author. Tel: +44-1223-332711. Fax: +441223-332662. be done over admissible state feedback policies, rather than open-loop input sequences, otherwise infeasibility and instability problems can occur [24]. However, optimization over arbitrary (nonlinear) feedback policies is particularly difficult if constraints have to be satisfied. Current proposals for achieving this using finite dimensional optimization, such as [26], are computationally intractable since the size of the optimization problem grows exponentially with the size of the problem data.

Hence, a popular approach in the predictive control literature is to compute one or more stabilizing linear state feedback control laws off-line and restrict the on-line computation to the selection of one of these control laws, followed by the computation of a finite sequence of admissible perturbations to the selected control law $[1,6$, 19,25]. Though this approach considerably reduces the computational complexity, it is not always obvious how to best choose the linear control laws off-line so as to minimize conservativeness.

An obvious improvement to this approach of "prestabilization" is to try to simultaneously compute the linear feedback control law and perturbation sequence on-line at each sample instant. However, the problem 
with this approach is that the predicted input and state sequences are nonlinear functions of the sequence of state feedback gains. As a consequence, the set of feasible decision variables is non-convex, in general. Various proposals have been put forward for modifying this problem so that the set of feasible decision variables is convex $[12,18,27]$, but generally this comes with an increase in the conservativeness of the solution. Hence, an interesting question is whether one can re-parameterize the optimal control problem, where the optimization is over the class of affine state feedback policies (linear feedback plus perturbation), and formulate an equivalent, but convex and tractable optimization problem. One of the contributions of this paper is to show that this is possible.

To show this, we exploit a recent result for solving a class of robust optimization problems with hard constraints, called adjustable robust counterpart (ARC) problems [3, 11 , where the optimization variables correspond to decisions that can be made once actual values of the uncertainty become available. The authors of $[3,11]$ proposed that, instead of solving for an admissible nonlinear function of the uncertainty, one could aim to parameterize the solution as an affine function of the uncertainty. They proceeded to show that if the uncertainty set is polyhedral and the constraints in the optimization problem are affine, then an affine function of the uncertainty can be found by solving a single, computationally tractable linear program (LP). Equivalent parameterizations have also been proposed in the literature on predictive control with Gaussian state disturbances [28] and bounded state disturbances [21]. This paper also presents a number of novel system-theoretic results relating to the use of this new parameterization.

In order for the results in this paper to be applicable to a large class of problems, the development of this paper starts with a general problem description, which is refined in each section. Section 2 discusses the class of systems that is to be considered throughout the paper and lists a number of standing assumptions. Section 3 describes the well-known affine state feedback parameterization and Section 4 describes the new affine disturbance feedback parameterization that was proposed in $[3,9,11,21,28]$.

We demonstrate that in the case of the state feedback parameterization of Section 3, the set of decisions variables is non-convex, in general. In contrast, with the disturbance feedback parameterization of Section 4, the set of admissible decision variables is convex, and an admissible policy can be found for a broad class of disturbances by solving a single and tractable convex optimization problem.

In Section 5 we state our main result and show that the state and disturbance feedback parameterizations of Sections 3 and 4 are equivalent. This has important system-theoretical consequences, which are explored in detail in Sections 6 and 7. Section 6 is concerned with results that guarantee robust constraint satisfaction for all time when the proposed control policy is used in the implementation of time-varying, receding horizon or time-optimal control laws. Section 7 formulates a suitable robust finite horizon optimal control problem and presents results that guarantee robust stability of the closed-loop system for the case when the solution to the optimal control problem is implemented in a receding horizon fashion. The paper concludes in Section 8 and suggests some topics for further research.

Notation and definitions: For matrices $A$ and $B$, let $A \leq B$ denote element-wise inequality. A matrix, not necessarily square, is referred to as (strictly) lower triangular if the $(i, j)$ entry is zero for all $i<j(i \leq j)$. A block partitioned matrix is referred to as (strictly) block lower triangular if the $(i, j)$ block is zero when $i<j(i \leq j)$; note that a block lower triangular matrix is not necessarily lower triangular. Given a vector $x,\|x\|_{A}^{2}:=x^{T} A x$. $\mathbb{Z}_{[k, l]}$ represents the set of integers $\{k, k+1, \ldots, l\}$. A continuous function $\gamma: \mathbb{R}_{\geq 0} \rightarrow \mathbb{R}_{\geq 0}$ is a $\mathcal{K}$-function if it is strictly increasing and $\gamma(0)=\overline{0}$; it is a $\mathcal{K}_{\infty}$-function if, in addition, $\gamma(s) \rightarrow \infty$ as $s \rightarrow \infty$. A continuous function $\beta: \mathbb{R}_{\geq 0} \times \mathbb{R}_{\geq 0} \rightarrow \mathbb{R}_{\geq 0}$ is a $\mathcal{K} \mathcal{L}$-function if for all $k \geq 0$, the function $\beta(\cdot, k)$ is a $\mathcal{K}$-function and for each $s \geq 0, \beta(s, \cdot)$ is decreasing with $\beta(s, k) \rightarrow 0$ as $k \rightarrow \infty$.

\section{Standing Assumptions}

Consider the following discrete-time LTI system:

$$
x^{+}=A x+B u+w,
$$

where $x \in \mathbb{R}^{n}$ is the system state at the current time instant, $x^{+}$is the state at the next time instant, $u \in \mathbb{R}^{m}$ is the control input and $w \in \mathbb{R}^{n}$ is the disturbance. The current and future values of the disturbance are unknown and may change unpredictably from one time instant to the next, but are contained in a convex and compact (closed and bounded) set $W$, which contains the origin. The actual values of the state, input and disturbance at time instant $k$ are denoted by $x(k), u(k)$ and $w(k)$, respectively; where it is clear from the context, $x$ will be used to denote the current value of the state. It is assumed that $(A, B)$ is stabilizable and that at each sample instant a measurement of the state is available. We also assume that a linear state feedback gain matrix $K \in \mathbb{R}^{m \times n}$ is given, such that $A+B K$ is strictly stable.

The system is subject to mixed constraints on the state and input:

$$
Z:=\left\{(x, u) \in \mathbb{R}^{n} \times \mathbb{R}^{m} \mid C x+D u \leq b\right\},
$$

where the matrices $C \in \mathbb{R}^{s \times n}, D \in \mathbb{R}^{s \times m}$ and the vector $b \in \mathbb{R}^{s}$. It is assumed that $Z$ is bounded and contains the 
origin in its interior. A primary design goal is to guarantee that the state and input of the closed-loop system remain in $Z$ for all time and for all allowable disturbance sequences. Finally, a target/terminal constraint set $X_{f}$ is given by

$$
X_{f}:=\left\{x \in \mathbb{R}^{n} \mid Y x \leq z\right\},
$$

where the matrix $Y \in \mathbb{R}^{r \times n}$ and the vector $z \in \mathbb{R}^{r}$. It is assumed that $X_{f}$ is bounded and contains the origin in its interior.

Remark 1 Many of the results in this paper remain valid if the assumption that $Z$ and $X_{f}$ are polytopes is relaxed to $Z$ and $X_{f}$ being convex; the current assumptions serve to simplify the presentation in Section 4.

In the sequel, predictions of the system's evolution over a finite control/planning horizon will be used to define a number of suitable control policies. Let the length $N$ of this planning horizon be a positive integer and define stacked versions of the predicted input, state and disturbance vectors $\mathbf{u} \in \mathbb{R}^{m N}, \mathbf{x} \in \mathbb{R}^{n(N+1)}$ and $\mathbf{w} \in \mathbb{R}^{n N}$, respectively, as

$$
\begin{aligned}
\mathbf{x} & :=\left[\begin{array}{lll}
x_{0}^{T} & \ldots & x_{N}^{T}
\end{array}\right]^{T} \\
\mathbf{u} & :=\left[\begin{array}{lll}
u_{0}^{T} & \ldots & u_{N-1}^{T}
\end{array}\right]^{T}, \\
\mathbf{w} & :=\left[\begin{array}{lll}
w_{0}^{T} & \ldots & w_{N-1}^{T}
\end{array}\right]^{T},
\end{aligned}
$$

where $x_{0}=x$ denotes the current measured value of the state and $x_{i+1}:=A x_{i}+B u_{i}+w_{i}, i=0, \ldots, N-1$ denote the prediction of the state after $i$ time instants. Finally, let the set $\mathcal{W}:=W^{N}:=W \times \cdots \times W$, so that $\mathbf{w} \in \mathcal{W}$.

\section{State Feedback Parameterization}

One natural approach to controlling the system in (1), while ensuring the satisfaction of the constraints, is to search over the set of time-varying affine state feedback control policies with knowledge of prior states:

$$
u_{i}=\sum_{j=0}^{i} L_{i, j} x_{j}+g_{i}, \quad \forall i \in \mathbb{Z}_{[0, N-1]}
$$

where each $L_{i, j} \in \mathbb{R}^{m \times n}$ and $g_{i} \in \mathbb{R}^{m}$. For notational convenience, we also define the block lower triangular matrix $\mathbf{L} \in \mathbb{R}^{m N \times n(N+1)}$ and stacked vector $\mathbf{g} \in \mathbb{R}^{m N}$ as

$$
\mathbf{L}:=\left[\begin{array}{cccc}
L_{0,0} & 0 & \cdots & 0 \\
\vdots & \ddots & \ddots & \vdots \\
L_{N-1,0} & \cdots & L_{N-1, N-1} & 0
\end{array}\right], \mathbf{g}:=\left[\begin{array}{c}
g_{0} \\
\vdots \\
g_{N-1},
\end{array}\right],
$$

so that the input sequence can be written as $\mathbf{u}=\mathbf{L x}+\mathbf{g}$. For a given initial state $x$, we say that the pair $(\mathbf{L}, \mathbf{g})$ is admissible if the control policy (4) guarantees that for all allowable disturbance sequences of length $N$, the constraints (2) are satisfied over the horizon $i=0, \ldots, N-1$ and that the state is in the target set (3) at the end of the horizon. More precisely, the set of admissible $(\mathbf{L}, \mathbf{g})$ is defined as

$$
\Pi_{N}^{s f}(x):=\left\{(\mathbf{L}, \mathbf{g}) \mid \begin{array}{r}
(\mathbf{L}, \mathbf{g}) \text { satisfies }(5), x=x_{0} \\
x_{i+1}=A x_{i}+B u_{i}+w_{i} \\
u_{i}=\sum_{j=0}^{i} L_{i, j} x_{j}+g_{i} \\
\left(x_{i}, u_{i}\right) \in Z, x_{N} \in X_{f} \\
\forall i \in \mathbb{Z}_{[0, N-1]}, \forall \mathbf{w} \in \mathcal{W}
\end{array}\right\}
$$

The set of initial states $x$ for which an admissible control policy of the form (4) exists is defined as

$$
X_{N}^{s f}:=\left\{x \in \mathbb{R}^{n} \mid \Pi_{N}^{s f}(x) \neq \emptyset\right\} .
$$

It is critical to note that it may not be possible to select a single $(\mathbf{L}, \mathbf{g})$ such that it is admissible for all $x \in X_{N}^{s f}$. Indeed, it is easy to find examples where there exists a pair $(x, \tilde{x}) \in X_{N}^{s f} \times X_{N}^{s f}$ such that $\Pi_{N}^{s f}(x) \bigcap_{N}^{s f}(\tilde{x})=\emptyset$. For problems of non-trivial size, it is therefore necessary to calculate an admissible pair $(\mathbf{L}, \mathbf{g})$ on-line, given a measurement of the current state $x$.

Once an admissible control policy is computed for the current state, there are many ways in which it can be applied to the system; time-varying, time-optimal and receding-horizon implementations are the most common, and are considered in detail in Sections 6 and 7.

Remark 2 Note that the state feedback policy (4) subsumes the well-known class of "pre-stabilizing" control policies [1,6,19,25], in which the control policy takes the form $u_{i}=K x_{i}+c_{i}$, where $K$ is computed off-line and on-line computation is limited to finding an admissible perturbation sequence $\left\{c_{i}\right\}_{i=0}^{N-1}$.

Finding an admissible pair $(\mathbf{L}, \mathbf{g})$, given the current state $x$, has been believed to be a very difficult problem due to the following property:

Proposition 3 (Non-convexity) For a given state $x \in X_{N}^{s f}$, the set of admissible affine state feedback control parameters $\Pi_{N}^{s f}(x)$ is non-convex, in general.

The truth of this statement is easily verified by considering the following example:

Example 4 Consider the SISO system $x^{+}=x+u+$ $w$ with initial state $x_{0}=0$, input constraint $|u| \leq 3$, bounded disturbances $|w| \leq 1$ and a planning horizon of 
$N=3$. Consider a control policy of the form (4) with $\mathbf{g}=0$ and $L_{2,1}=0$, so that $u_{0}=0$ and

$$
\begin{aligned}
& u_{1}=L_{1,1} w_{0} \\
& u_{2}=\left[L_{2,2}\left(1+L_{1,1}\right)\right] w_{0}+L_{2,2} w_{1}
\end{aligned}
$$

Since the constraints on the components of $\mathbf{w}$ are independent, it is easy to show that the input constraints are satisfied for all $\mathbf{w} \in \mathcal{W}$ if and only if

$$
\begin{aligned}
\left|L_{1,1}\right| & \leq 3 \\
\left|L_{2,2}\left(1+L_{1,1}\right)\right|+\left|L_{2,2}\right| & \leq 3 .
\end{aligned}
$$

It is straightforward to verify that the set of gains $\left(L_{1,1}, L_{2,2}\right)$ that satisfy these constraints is non-convex; the pairs $(-3,1)$ and $(-1,3)$ are admissible, while the pair $(-2,2)$ is not.

It is surprising to note that, though the set $\Pi_{N}^{s f}(x)$ may be non-convex, the set $X_{N}^{s f}$ is always convex. We defer the proof of this until Section 5. Additionally, despite the fact that $\Pi_{N}^{s f}(x)$ may be non-convex, we will show that one can still find an admissible $(\mathbf{L}, \mathbf{g})$ by solving a single tractable convex programming problem.

\section{Disturbance Feedback Parameterization}

An alternative to (4) is to parameterize the control policy as an affine function of the sequence of past disturbances, so that

$$
u_{i}=\sum_{j=0}^{i-1} M_{i, j} w_{j}+v_{i}, \quad \forall i \in \mathbb{Z}_{[0, N-1]},
$$

where each $M_{i, j} \in \mathbb{R}^{m \times n}$ and $v_{i} \in \mathbb{R}^{m}$. It should be noted that, since full state feedback is assumed, the past disturbance sequence is easily calculated as the difference between the predicted and actual states at each step, i.e.

$$
w_{i}=x_{i+1}-A x_{i}-B u_{i}, \quad \forall i \in \mathbb{Z}_{[0, N-1]} .
$$

The above parameterization appears to have originally been suggested some time ago within the context of stochastic programs with recourse [9]. More recently, it has been revisited as a means for finding solutions to a class of robust optimization problems, called affinely adjustable robust counterpart (AARC) problems [3,11], and robust model predictive control problems $[21,28]$.

For notational convenience, we define the vector $\mathbf{v} \in$ $\mathbb{R}^{m N}$ and the strictly block lower triangular matrix $\mathbf{M} \in$
$\mathbb{R}^{m N \times n N}$ such that

$$
\mathbf{M}:=\left[\begin{array}{cccc}
0 & \cdots & \cdots & 0 \\
M_{1,0} & 0 & \ldots & 0 \\
\vdots & \ddots & \ddots & \vdots \\
M_{N-1,0} & \cdots & M_{N-1, N-2} & 0
\end{array}\right], \mathbf{v}:=\left[\begin{array}{c}
v_{0} \\
\vdots \\
\vdots \\
v_{N-1}
\end{array}\right]
$$

so that the input sequence can be written as $\mathbf{u}=\mathbf{M w}+$ v. In a manner similar to (6), we define the set of admissible $(\mathbf{M}, \mathbf{v})$ as

$$
\Pi_{N}^{d f}(x):=\left\{(\mathbf{M}, \mathbf{v}) \mid \begin{array}{r}
(\mathbf{M}, \mathbf{v}) \text { satisfies }(10), x=x_{0} \\
x_{i+1}=A x_{i}+B u_{i}+w_{i} \\
u_{i}=\sum_{j=0}^{i-1} M_{i, j} w_{j}+v_{i} \\
\left(x_{i}, u_{i}\right) \in Z, x_{N} \in X_{f} \\
\forall i \in \mathbb{Z}_{[0, N-1]}, \forall \mathbf{w} \in \mathcal{W}
\end{array}\right\}
$$

and the set of initial states $x$ for which an admissible control policy of the form (8) exists as

$$
X_{N}^{d f}:=\left\{x \in \mathbb{R}^{n} \mid \Pi_{N}^{d f}(x) \neq \emptyset\right\} .
$$

Before proceeding, we note that one can easily find matrices $F \in \mathbb{R}^{t \times m N}, G \in \mathbb{R}^{t \times n N}, H \in \mathbb{R}^{t \times n}$ and a vector $c \in \mathbb{R}^{t}$, where $t:=s N+r$ (for completeness, the matrices and vectors are given in the Appendix), such that the expression for $\Pi_{N}^{d f}(x)$ can be rewritten more compactly as

$\Pi_{N}^{d f}(x)=\left\{\begin{array}{l|r}(\mathbf{M}, \mathbf{v}) & \begin{array}{r}(\mathbf{M}, \mathbf{v}) \text { satisfies }(10) \\ F \mathbf{v}+(F \mathbf{M}+G) \mathbf{w} \leq c+H x \\ \forall \mathbf{w} \in \mathcal{W}\end{array}\end{array}\right\}$.

\subsection{Convexity of $\Pi_{N}^{d f}(x)$}

The main advantage of the disturbance feedback parameterization in (8) over the state feedback parameterization in (4) is formalized in the following statement:

Proposition 5 (Convexity) For a given state $x \in$ $X_{N}^{d f}$, the set of admissible affine disturbance feedback parameters $\Pi_{N}^{d f}(x)$ is convex and closed. Furthermore, the set of states $X_{N}^{d f}$, for which at least one admissible affine disturbance feedback policy exists, is convex.

PROOF. Consider the set

$$
\mathcal{C}_{N}:=\left\{\begin{array}{r|r}
(\mathbf{M}, \mathbf{v}) \text { satisfies }(10) \\
(\mathbf{M}, \mathbf{v}, x) & F \mathbf{v}+(F \mathbf{M}+G) \mathbf{w} \leq c+H x \\
\forall \mathbf{w} \in \mathcal{W}
\end{array}\right\} .
$$


It immediately follows that

$\mathcal{C}_{N}=\bigcap_{\mathbf{w} \in \mathcal{W}}\left\{(\mathbf{M}, \mathbf{v}, x) \mid \begin{array}{r}(\mathbf{M}, \mathbf{v}) \text { satisfies }(10) \\ F \mathbf{v}+(F \mathbf{M}+G) \mathbf{w} \leq c+H x\end{array}\right\}$

$\mathcal{C}_{N}$ is closed and convex, since it is the intersection of an arbitrary collection of closed and convex sets. The set $X_{N}^{d f}$ is convex since it is a projection of $\mathcal{C}_{N}$ onto a suitably-defined subspace. Since the set $\Pi_{N}^{d f}(x)$ in (13) can similarly be written as an intersection of closed and convex sets, it is also closed and convex.

The above result is of fundamental importance. If $\mathcal{W}$ is convex and compact, then it is conceptually possible to compute a pair $(\mathbf{M}, \mathbf{v}) \in \Pi_{N}^{d f}(x)$ in a computationally tractable way, given the current state $x$.

Remark 6 Note that the proof of Proposition 5 does not require $\mathcal{W}$ to be convex. However, the set $\mathcal{W}$ can, without loss of generality or increased conservativeness, be replaced by its convex hull; see [8, Ex. 7.1.2].

\subsection{Computation of Admissible Policies}

It is well-known that one can eliminate the universal quantifier in (13) to obtain the equivalent expression

$\Pi_{N}^{d f}(x)=\left\{(\mathbf{M}, \mathbf{v}) \mid \begin{array}{r}(\mathbf{M}, \mathbf{v}) \text { satisfies }(10) \\ F \mathbf{v}+\max _{\mathbf{w} \in \mathcal{W}}(F \mathbf{M}+G) \mathbf{w} \leq c+H x\end{array}\right\}$,

where $\max _{\mathbf{w} \in \mathcal{W}}(F \mathbf{M}+G) \mathbf{w}$ denotes row-wise maximization. When $\mathcal{W}$ is convex, computing an admissible policy is easily done by formulating the dual of each maximization problem $\max _{\mathbf{w} \in \mathcal{W}}(F \mathbf{M}+G)_{i} \mathbf{w}, i=1, \ldots, t$, introducing some slack variables and solving a single, suitably-defined convex programming problem, where $(F \mathbf{M}+G)_{i}$ represent the $i^{\text {th }}$ row of $(F \mathbf{M}+G)$. We provide two examples for commonly encountered disturbance sets:

Example 7 (Polytopic Disturbance Sets [3, 11]) Suppose that $\mathcal{W}$ is a polytope (closed and bounded polyhedron). In this case the disturbance set may be written as

$$
\mathcal{W}=\left\{\mathbf{w} \in \mathbb{R}^{n N} \mid S \mathbf{w} \leq h\right\}
$$

where $S \in \mathbb{R}^{a \times n}$ and $h \in \mathbb{R}^{a}$. Note that this includes cases where the disturbance set is time-varying, and that both 1 - and $\infty$-norm disturbance sets $W$ can be characterized in this manner.

In this case, each row of $\max _{\mathbf{w} \in \mathcal{W}}(F \mathbf{M}+G) \mathbf{w}$ is actually an LP, and exploitation of its dual leads to a constraint of the form

$$
\begin{gathered}
\max _{\mathbf{w} \in \mathcal{W}}(F \mathbf{M}+G)_{i} \mathbf{w}=\min _{\mathbf{z}_{i}} h^{T} \mathbf{z}_{i}, \\
\text { s.t. } S^{T} \mathbf{z}_{i}=(F \mathbf{M}+G)_{i}^{T}, \mathbf{z}_{i} \geq 0
\end{gathered}
$$

where the vector $\mathbf{z}_{i} \in \mathbb{R}^{a}$ represents the dual variables associated with the $i^{\text {th }}$ row of the maximization in (14). By combining these into a matrix $\mathbf{Z}:=\left[\begin{array}{lll}\mathbf{z}_{1} & \ldots & \mathbf{z}_{N}\end{array}\right] \in \mathbb{R}^{a \times t}$, one can rewrite $\Pi_{N}^{d f}(x)$ in terms of purely affine constraints:

$$
\Pi_{N}^{d f}(x)=\left\{(\mathbf{M}, \mathbf{v}) \mid \begin{array}{r}
(\mathbf{M}, \mathbf{v}) \text { satisfies }(10), \exists \mathbf{Z} \text { s.t. } \\
F \mathbf{v}+\mathbf{Z}^{T} h \leq c+H x \\
F \mathbf{M}+G=\mathbf{Z}^{T} S, \mathbf{Z} \geq 0
\end{array}\right\}
$$

where all inequalities are element-wise. An admissible pair $(\mathbf{M}, \mathbf{v})$ can then be found by solving a single LP in a polynomial number of decision variables and constraints. Note that standard Kronecker product identities can be used to convert the matrix products in (15) to a vectorized form compatible with standard LP formulations.

Example 8 (Norm-Bounded Disturbance Sets)

Suppose that $\mathcal{W}$ represents the affine map of a set of norm-bounded signals:

$$
\mathcal{W}=\left\{\mathbf{w} \mid \mathbf{w}=E \mathbf{d}+f, \quad\|\mathbf{d}\|_{p} \leq 1\right\}
$$

where $E \in \mathbb{R}^{n N \times l}$ and $f \in \mathbb{R}^{n N}$. From the well-known properties of the dual norm, it immediately follows that

$$
\max _{\mathbf{w} \in \mathcal{W}} a^{T} \mathbf{w}=\left\|E^{T} a\right\|_{q}+a^{T} f
$$

for any vector $a \in \mathbb{R}^{n N}$, where $1 / p+1 / q=1$. In particular, if $\mathcal{W}$ is the linear map of a 2 -norm ball, then the disturbance set (with $f=0, p=q=2$ ) can be written as $\mathcal{W}=\left\{\right.$ E $\left.\mathbf{d} \mid\|\mathbf{d}\|_{2} \leq 1\right\}$. The row-wise maximization in (14) can then be simplified to

$\Pi_{N}^{d f}(x)=\left\{\begin{array}{l|r}(\mathbf{M}, \mathbf{v}) & \begin{array}{r}(\mathbf{M}, \mathbf{v}) \text { satisfies }(10) \\ F_{i} \mathbf{v}+\left\|(F \mathbf{M}+G)_{i} E\right\|_{2} \leq c_{i}+H_{i} x \\ \forall i \in \mathbb{Z}_{[1, t]}\end{array}\end{array}\right\}$

where $F_{i}, c_{i}$, and $H_{i}$ represent the $i^{\text {th }}$ rows of the respective matrices. An admissible pair $(\mathbf{M}, \mathbf{v})$ can then be found by solving a single second-order cone program (SOCP) in a polynomial number of variables and constraints [20]. A similar process leads, in the case of 1 - or $\infty$-norm bounded disturbances, to a tractable LP. Alternatively, these may be handled as special cases of Example $\%$. 


\section{Equivalence between State and Disturbance Feedback Parameterizations}

One important question is whether the disturbance feedback parameterization (8) is more or less conservative than the state feedback parameterization (4). We next show that they are actually equivalent:

Theorem 9 (Equivalence) The set of admissible states $X_{N}^{d f}=X_{N}^{s f}$. Additionally, given any $x \in X_{N}^{s f}$, for any admissible $(\mathbf{L}, \mathbf{g})$ an admissible $(\mathbf{M}, \mathbf{v})$ can be found that yields the same state and input sequence for all allowable disturbance sequences, and vice-versa.

PROOF. $X_{N}^{s f} \subseteq X_{N}^{d f}$ : By definition, for a given $x \in$ $X_{N}^{s f}$, there exists a pair $(\mathbf{L}, \mathbf{g})$ that satisfies the constraints in (6). For a given disturbance sequence $\mathbf{w} \in \mathcal{W}$, the inputs and states of the system can be written as :

$$
\mathbf{u}=\mathbf{L} \mathbf{x}+\mathbf{g}, \quad \mathbf{x}=(I-\mathbf{B L})^{-1}(\mathbf{B g}+\mathbf{E w}+\mathbf{A} x)
$$

where the matrices $\mathbf{A}, \mathbf{B}$, and $\mathbf{E}$ (for completeness, these are given in the Appendix) are defined so that one can write $\mathbf{x}=\mathbf{A} x+\mathbf{B u}+\mathbf{E w}$. The matrix $I-\mathbf{B L}$ is always non-singular, since $\mathbf{B L}$ is strictly lower triangular. The control sequence can then be rewritten as $\mathbf{u}=\mathbf{L}(I-\mathbf{B L})^{-1}(\mathbf{B g}+\mathbf{A} x)+\mathbf{L}(I-\mathbf{B L})^{-1} \mathbf{E} \mathbf{w}+\mathbf{g}$, and an admissible $(\mathbf{M}, \mathbf{v})$ constructed by choosing

$$
\begin{aligned}
\mathbf{M} & =\mathbf{L}(I-\mathbf{B L})^{-1} \mathbf{E} \\
\mathbf{v} & =\mathbf{L}(I-\mathbf{B L})^{-1}(\mathbf{B g}+\mathbf{A} x)+\mathbf{g} .
\end{aligned}
$$

It is easy to verify that this (M, v) satisfies (11), and gives exactly the same input sequence as the pair $(\mathbf{L}, \mathbf{g})$. Therefore, $(\mathbf{M}, \mathbf{v}) \in \Pi_{N}^{d f}(x)$, thus $x \in X_{N}^{s f} \Rightarrow x \in X_{N}^{d f}$.

$X_{N}^{d f} \subseteq X_{N}^{s f}$ : By definition, for a given $x \in X_{N}^{d f}$, there exists a pair $(\mathbf{M}, \mathbf{v})$ that satisfies the constraints in (11). For a given disturbance sequence $\mathbf{w} \in \mathcal{W}$, the inputs and states of the system can be written as:

$$
\mathbf{u}=\mathbf{M w}+\mathbf{v}, \quad \mathbf{x}=\mathbf{B}(\mathbf{M w}+\mathbf{v})+\mathbf{E w}+\mathbf{A} x
$$

Recall that since full state feedback is assumed, one can construct matrices $\mathbf{E}^{\dagger}$ and $\mathcal{I}$ such that the disturbances can be recovered using $\mathbf{w}=\mathbf{E}^{\dagger} \mathbf{x}-\mathcal{I} A x+\mathbf{E}^{\dagger} \mathbf{B u}$. It is easy to verify that the matrices $\mathbf{E}^{\dagger}$ and $\mathcal{I}^{T}$ are left inverses of $\mathbf{E}$ and $\mathbf{A}$ respectively, so that $\mathbf{E}^{\dagger} \mathbf{E}=I$ and $\mathcal{I}^{T} \mathbf{A}=I$. The input sequence can then be rewritten as $\mathbf{u}=(I-$ $\left.\mathbf{M E}^{\dagger} \mathbf{B}\right)^{-1}\left(\mathbf{M} \mathbf{E}^{\dagger} \mathbf{x}-\mathbf{M} \mathcal{I} A x+\mathbf{v}\right)$. The matrix $I-\mathbf{M} \mathbf{E}^{\dagger} \mathbf{B}$ is non-singular because the product $\mathbf{M} \mathbf{E}^{\dagger} \mathbf{B}=\mathbf{M}(I \otimes B)$ is strictly lower triangular. An admissible $(\mathbf{L}, \mathbf{g})$ can then be constructed by choosing

$$
\begin{aligned}
& \mathbf{L}=\left(I-\mathbf{M} \mathbf{E}^{\dagger} \mathbf{B}\right)^{-1} \mathbf{M} \mathbf{E}^{\dagger} \\
& \mathbf{g}=\left(I-\mathbf{M} \mathbf{E}^{\dagger} \mathbf{B}\right)^{-1}(\mathbf{v}-\mathbf{M} \mathcal{I} A x)
\end{aligned}
$$

It is easy to verify that this $(\mathbf{L}, \mathbf{g})$ satisfies (6), and gives exactly the same input sequence as the pair $(\mathbf{M}, \mathbf{v})$. Therefore, $(\mathbf{L}, \mathbf{g}) \in \Pi_{N}^{s f}(x)$, thus $x \in X_{N}^{d f} \Rightarrow x \in X_{N}^{s f}$.

This leads to the following surprising result:

Corollary 10 (Convexity of $X_{N}^{s f}$ ) The set of states $X_{N}^{s f}$, for which an admissible affine state feedback policy of the form (4) exists, is a convex set.

\section{Geometric and Invariance Properties}

It is well-known that the set of states for which an admissible open loop input sequence exists (i.e. a feedback policy with $\mathbf{L}=0$ or $\mathbf{M}=0$ ) may collapse to the empty set if the horizon is sufficiently large [26, Sect. F]. Furthermore, for time-varying, receding-horizon or timeoptimal control implementations, it may not be possible to guarantee constraint satisfaction for all time unless additional assumptions are made. In this section, we provide conditions under which these problems will not occur. We first introduce the following standard assumption (cf. [24]):

A1 (Terminal constraint) The state feedback gain matrix $K$ and terminal constraint $X_{f}$ have been chosen such that:

- $X_{f}$ is contained inside the set of states for which the constraints (2) are satisfied under the control $u=K x$, i.e. $X_{f} \subseteq\{x \mid(x, K x) \in Z\}=\{x \mid(C+D K) x \leq b\}$.

- $X_{f}$ is robust positively invariant for the closed-loop system $x^{+}=(A+B K) x+w$, i.e. $(A+B K) x+w \in X_{f}$, for all $x \in X_{f}$ and all $w \in W$.

Under some additional, mild technical assumptions, one can compute a $K$ and a polytopic $X_{f}$ that satisfies A1 if $W$ is a polytope, an ellipsoid or the affine map of a $p$-norm ball. The reader is referred to $[5,17,19]$ and the references therein for details.

\subsection{Monotonicity of $X_{N}^{s f}$ and $X_{N}^{d f}$}

We are now in a position to give a sufficient condition under which one can guarantee that $X_{N}^{s f}$ (equivalently, $\left.X_{N}^{d f}\right)$ is non-empty and the size of $X_{N}^{s f}$ is non-decreasing (with respect to set inclusion) with horizon length $N$ :

Proposition 11 (Size of $X_{N}^{s f}$ and $X_{N}^{s f}$ ) If $\boldsymbol{A} 1$ holds, then the following set inclusions hold:

$$
\begin{aligned}
& X_{f} \subseteq X_{1}^{s f} \subseteq \cdots \subseteq X_{N-1}^{s f} \subseteq X_{N}^{s f} \subseteq X_{N+1}^{s f} \subseteq \cdots \\
& X_{f} \subseteq X_{1}^{d f} \subseteq \cdots \subseteq X_{N-1}^{d f} \subseteq X_{N}^{d f} \subseteq X_{N+1}^{d f} \subseteq \cdots
\end{aligned}
$$


PROOF. The proof of the first relation is by induction. Let $x \in X_{N}^{s f}$ and $(\mathbf{L}, \mathbf{g}) \in \Pi_{N}^{s f}(x)$. One can construct a pair $(\overline{\mathbf{L}}, \overline{\mathbf{g}}) \in X_{N+1}^{s f}$, where $\overline{\mathbf{L}}:=\left[\begin{array}{lll}\mathbf{L} & 0 & 0 \\ 0 & K & 0\end{array}\right]$ and $\overline{\mathbf{g}}:=$ $\left[\begin{array}{ll}\mathbf{g}^{T} & 0\end{array}\right]^{T}$, such that the final stage input will be $u_{N}=$ $K x_{N}$. From the definition of $\Pi_{N}^{s f}(x)$, it follows that $x_{N} \in$ $X_{f}$. If A1 holds, then $\left(x_{N}, u_{N}\right) \in Z$ and $x_{N+1}=A x_{N}+$ $B u_{N}+w_{N} \in X_{f}$ for all $w_{N} \in W$. It then follows from the definition of $\Pi_{N+1}^{s f}(x)$ that $(\overline{\mathbf{L}}, \overline{\mathbf{g}}) \in \Pi_{N+1}^{s f}(x)$, hence $x \in X_{N+1}^{s f}$. The proof is completed by verifying, in a similar manner, that $X_{f} \subseteq X_{1}^{s f} \subseteq X_{2}^{s f}$. The second relation then follows from Theorem 9 .

\subsection{Time-varying Control Laws}

We first consider what happens if one were to implement an admissible affine disturbance feedback policy in a time-varying fashion. Given any $(\mathbf{M}, \mathbf{v}) \in \Pi_{N}^{d f}(x(0))$, we consider the following time-varying affine disturbance feedback policy:

$$
u(k)= \begin{cases}v_{k}+\sum_{j=0}^{k-1} M_{i, j} w(j) & \text { if } k \in \mathbb{Z}_{[0, N-1]} \\ K x(k) & \text { if } k \in \mathbb{Z}_{[N, \infty)}\end{cases}
$$

Recall that the realized disturbance sequence $w(\cdot)$ can be recovered using the relation (9). Theorem 9 implies that we could also have defined an equivalent, time-varying affine state feedback policy, but we choose to work with disturbance feedback policies due to the convenience of computation resulting from Proposition 5 and Examples 7 and 8 . The next result follows immediately:

Proposition 12 (Time-varying control) Let A1 hold, the initial state $x(0) \in X_{N}^{d f}$ and $(\mathbf{M}, \mathbf{v}) \in$ $\Pi_{N}^{d f}(x(0))$. For all allowable infinite disturbance sequences, the state of system (1), in closed-loop with the feedback policy (18), enters $X_{f}$ in $N$ steps or less and is in $X_{f}$ for all $k \in \mathbb{Z}_{[N, \infty)}$. Furthermore, the constraints in (2) are satisfied for all time and for all allowable infinite disturbance sequences.

\subsection{Receding Horizon Control Laws}

We next consider what happens when the disturbance feedback parameterization (8) is used to design a receding horizon control (RHC) law. In RHC, an admissible feedback policy is computed at each time instant, but only the first component of the policy is applied.

Consider the set-valued map $\kappa_{N}: X_{N}^{s f} \rightarrow 2^{\mathbb{R}^{m}}\left(2^{\mathbb{R}^{m}}\right.$ is the set of all subsets of $\mathbb{R}^{m}$ ), which is defined by considering only the first portion of an admissible state feedback parameter $(\mathbf{L}, \mathbf{g})$, i.e.

$$
\kappa_{N}(x):=\left\{u \mid \exists(\mathbf{L}, \mathbf{g}) \in \Pi_{N}^{s f}(x) \text { s.t. } u=L_{0,0} x+g_{0}\right\} .
$$

An admissible RHC law $\mu_{N}: X_{N}^{s f} \rightarrow \mathbb{R}^{m}$ is defined as any selection from the set-valued map $\kappa_{N}(\cdot)$, i.e. $\mu_{N}(\cdot)$ has to satisfy $\mu_{N}(x) \in \kappa_{N}(x)$ for all $x \in X_{N}^{s f}$. The resulting closed-loop system is then given by

$$
x^{+}=A x+B \mu_{N}(x)+w .
$$

Note that the RHC law $\mu_{N}(\cdot)$ is time-invariant and is, in general, a nonlinear function of the current state. Due to the non-convexity of $\Pi_{N}^{s f}(x)$, computing an admissible $(\mathbf{L}, \mathbf{g})$ in (19) at each time instant is problematic. However, by a straightforward application of Theorem 9 , it follows that

$$
\kappa_{N}(x)=\left\{u \mid \exists(\mathbf{M}, \mathbf{v}) \in \Pi_{N}^{d f}(x) \text { s.t. } u=v_{0}\right\} .
$$

Hence, computation of a $\mu_{N}(x) \in \kappa_{N}(x)$ is possible using convex programming methods.

The following result is easily proven using standard methods in RHC [24], by employing the state feedback parameterization (4):

Proposition 13 (RHC) If $\boldsymbol{A} 1$ holds, then the set $X_{N}^{s f}$ is robust positively invariant for the closed-loop system (20), i.e. if $x \in X_{N}^{s f}$, then $A x+B \mu_{N}(x)+w \in X_{N}^{s f}$ for all $w \in W$. Furthermore, the constraints (2) are satisfied for all time and for all allowable disturbance sequences if and only if the initial state $x(0) \in X_{N}^{s f}$.

\subsection{Minimum-time Control Laws}

We conclude this section by deriving some results for robust minimum-time control laws. Given a maximum horizon length $N_{\max }$ and the set $\mathcal{N}:=\left\{1, \ldots, N_{\max }\right\}$, let

$$
N^{*}(x):=\min _{N}\left\{N \in \mathcal{N} \mid \Pi_{N}^{s f}(x) \neq \emptyset\right\}
$$

be the minimum horizon length for which an admissible affine state feedback policy of the form (4) exists. Consider also the set-valued map $\kappa: \mathcal{X} \rightarrow 2^{\mathbb{R}^{m}}$, defined as

$$
\kappa(x):= \begin{cases}\kappa_{N^{*}(x)}(x) & \text { if } x \notin X_{f} \\ K x & \text { if } x \in X_{f}\end{cases}
$$

where $\kappa_{N^{*}(x)}(x)$ is defined as in (19) with $N=N^{*}(x)$, and

$$
\mathcal{X}:=X_{f} \cup\left(\cup_{N \in \mathcal{N}} X_{N}^{s f}\right) .
$$

Let the time-invariant robust time-optimal control law $\mu: \mathcal{X} \rightarrow \mathbb{R}^{m}$ be any selection from $\kappa(\cdot)$, i.e. $\mu(x) \in \kappa(x)$, for all $x \in \mathcal{X}$. Note that $\kappa(\cdot)$ is defined everywhere on $\mathcal{X}$ and that the state of the closed-loop system $x^{+}=$ $A x+B \mu(x)+w$ will enter $X_{f}$ in less than $N_{\max }$ steps 
if this is possible, even if $\mathbf{A 1}$ does not hold. Proof of the following result is straightforward and closely parallels that of Propositions 11 and 13.

Proposition 14 (Minimum-time control) If $A 1$ holds, then $\mathcal{X}=X_{N_{\max }}^{s f}$ and $\mathcal{X}$ is robust positively invariant for the closed-loop system $x^{+}=A x+B \mu(x)+w$, i.e. if $x \in \mathcal{X}$, then $x^{+} \in \mathcal{X}$ for all $w \in W$. The state of the closed-loop system enters $X_{f}$ in $N_{\max }$ steps or less and, once inside, remains inside for all time and all allowable disturbance sequences. Furthermore, the constraints (2) are satisfied for all time and for all allowable disturbance sequences if and only if the initial state $x(0) \in \mathcal{X}$.

\section{Uniqueness, Continuity and Stability of a Class of Receding Horizon Control Laws}

We next consider the important problem of how to synthesize an RHC law such that the closed-loop system is robustly stable. We choose to minimize the value of a cost function that is quadratic in the disturbance-free states and control inputs and demonstrate that this allows for the synthesis of a continuous RHC law, which guarantees that the closed-loop system is input-to-state stable (ISS). As in Section 6, we rely heavily on Theorem 9 in order to derive these results, moving freely between the two parameterizations and using whichever is most natural in each context.

\subsection{Cost Function}

We define an optimal policy to be one that minimizes the value of a cost function that is quadratic in the disturbance-free state and input sequences. We thus define:

$$
V_{N}(x, \mathbf{L}, \mathbf{g}, \mathbf{w}):=\sum_{i=0}^{N-1} \frac{1}{2}\left(\left\|\tilde{x}_{i}\right\|_{Q}^{2}+\left\|\tilde{u}_{i}\right\|_{R}^{2}\right)+\frac{1}{2}\left\|\tilde{x}_{N}\right\|_{P}^{2}
$$

where $\tilde{x}_{0}=x, \tilde{x}_{i+1}=A \tilde{x}_{i}+B \tilde{u}_{i}+w_{i}$ and $\tilde{u}_{i}=$ $\sum_{j=0}^{i} L_{i, j} \tilde{x}_{j}+g_{i}$ for $i=0, \ldots, N-1$, and $P, Q$ and $R$ are positive definite; and define an optimal policy pair as

$$
\left(\mathbf{L}^{*}(x), \mathbf{g}^{*}(x)\right):=\underset{(\mathbf{L}, \mathbf{g}) \in \Pi_{N}^{s f}(x)}{\operatorname{argmin}} V_{N}(x, \mathbf{L}, \mathbf{g}, 0) .
$$

The time-invariant receding horizon control law $\mu_{N}$ : $X_{N}^{s f} \rightarrow \mathbb{R}^{m}$ is defined by the first part of the optimal affine state feedback control policy, i.e.

$$
\mu_{N}(x):=L_{0,0}^{*}(x) x+g_{0}^{*}(x)
$$

which is nonlinear, in general. The closed-loop system becomes

$$
x^{+}=A x+B \mu_{N}(x)+w .
$$

We also define the value function $V_{N}^{*}: X_{N}^{s f} \rightarrow \mathbb{R}_{\geq 0}$ to be

$$
V_{N}^{*}(x):=\min _{(\mathbf{L}, \mathbf{g}) \in \Pi_{N}^{s f}(x)} V_{N}(x, \mathbf{L}, \mathbf{g}, 0)
$$

\subsection{Exploiting Equivalence to Compute the RHC Law}

For the equivalent affine disturbance feedback parameterization (8), we define a cost function $J_{N}(\cdot)$ analogous to the one defined in (21), i.e.

$$
J_{N}(x, \mathbf{M}, \mathbf{v}, \mathbf{w}):=\frac{1}{2} \sum_{i=0}^{N-1}\left(\left\|\bar{x}_{i}\right\|_{Q}^{2}+\left\|\bar{u}_{i}\right\|_{R}^{2}\right)+\frac{1}{2}\left\|\bar{x}_{N}\right\|_{P}^{2}
$$

where $\bar{x}_{0}=x, \bar{x}_{i+1}=A \bar{x}_{i}+B \bar{u}_{i}+w_{i}$ and $\bar{u}_{i}=$ $\sum_{j=0}^{i-1} M_{i, j} w_{j}+v_{i}$ for $i=0, \ldots, N-1$. If we define

$$
\left(\mathbf{M}^{*}(x), \mathbf{v}^{*}(x)\right):=\underset{(\mathbf{M}, \mathbf{v}) \in \Pi_{N}^{d f}(x)}{\operatorname{argmin}} J_{N}(x, \mathbf{M}, \mathbf{v}, 0)
$$

then the proof of the following result follows by a straightforward application of Theorem 9.

Proposition 15 (Computation of RHC law) The $R H C$ law $\mu_{N}(\cdot)$, defined in (23), is given by the first part of the optimal control sequence $\mathbf{v}^{*}(\cdot)$, i.e.

$$
\mu_{N}(x)=v_{0}^{*}(x)=L_{0,0}^{*}(x) x+g_{0}^{*}(x), \quad \forall x \in X_{N}^{s f} .
$$

The minimum value of $J_{N}(x, \cdot, \cdot, 0)$ taken over the set of admissible affine disturbance feedback parameters is equal to $V_{N}^{*}(x)$, defined in $(25)$, i.e.

$$
V_{N}^{*}(x)=\min _{(\mathbf{M}, \mathbf{v}) \in \Pi_{N}^{d f}(x)} J_{N}(x, \mathbf{M}, \mathbf{v}, 0) .
$$

Remark $16\left(\mathbf{L}^{*}(x), \mathbf{g}^{*}(x)\right)$ in $(22)$ is found by letting $(\mathbf{M}, \mathbf{v})=\left(\mathbf{M}^{*}(x), \mathbf{v}^{*}(x)\right)$ in $(17)$. This is important because it allows one to efficiently compute the value of the RHC law $u=\mu_{N}(x)$ via the minimization of a convex function over a convex set. In particular, we note that if $W$ is a polytope as in Example 7, then (26) can be written as a convex quadratic program (QP) in a tractable number of variables and constraints. If $W$ is an ellipsoid or the affine map of a Euclidean ball as in Example 8, then the optimization problem in (26) can be converted to a tractable SOCP [20].

\subsection{Continuity of the RHC Law and Value Function}

Proposition 17 (Continuity of $\mu_{N}$ and $V_{N}^{*}$ ) If $W$ is a polytope, then the receding horizon control law $\mu_{N}(\cdot)$ in (23) is unique and Lipschitz continuous on $X_{N}^{s f}$. Furthermore, the value function $V_{N}^{*}(\cdot)$ in $(25)$ is strictly convex and Lipschitz continuous on $X_{N}^{s f}$. 
PROOF. Note that $J_{N}(x, \mathbf{M}, \mathbf{v}, 0)=J_{N}(x, 0, \mathbf{v}, 0)$ for all $\mathbf{M}$. Hence, if we define the set

$$
\mathcal{V}_{N}(x):=\left\{\mathbf{v} \mid \exists \mathbf{M} \text { s.t. }(\mathbf{M}, \mathbf{v}) \in \Pi_{N}^{d f}(x)\right\}
$$

then, from (26) and Proposition 15 respectively,

$$
\begin{aligned}
\mathbf{v}^{*}(x) & =\underset{\mathbf{v} \in \mathcal{V}_{N}(x)}{\operatorname{argmin}} J_{N}(x, 0, \mathbf{v}, 0) \\
V_{N}^{*}(x) & =\min _{\mathbf{v} \in \mathcal{V}_{N}(x)} J_{N}(x, 0, \mathbf{v}, 0) .
\end{aligned}
$$

If $\mathcal{W}$ is a polytope, then $\mathcal{V}_{N}(x)$ is polyhedral since it is the projection of the polyhedron (15) onto a subspace. It is also easy to verify that $(x, \mathbf{v}) \mapsto J_{N}(x, 0, \mathbf{v}, 0)$ is a strictly convex quadratic function, and thus that (28) is a strictly convex QP. By applying the results in [2], it follows that $\mathbf{v}^{*}(\cdot)$ and hence $\mu_{N}(\cdot)$ are continuous, piecewise affine functions on $X_{N}^{s f}$, and that $V_{N}^{*}(\cdot)$ is a strictly convex, piecewise quadratic function on $X_{N}^{s f}$. Lipschitz continuity follows from the assumption that $Z$ is compact, hence $X_{N}^{s f}$ is also compact.

Finally, we present the following result, which is useful in proving stability in the next section:

Lemma 18 (Value at the origin) If $\boldsymbol{A} 1$ holds, then $V_{N}^{*}(0)=0$ and $\mu_{N}(0)=0$.

PROOF. Proposition 11 implies that the origin is in the interior of $X_{N}^{s f}$. If $x \in X_{f}$, then $(\mathbf{L}, \mathbf{g}) \in \Pi_{N}^{s f}(x)$ if $\mathbf{g}=0, L_{i, i}=K$ for $i=0, \ldots, N-1$ and $L_{i, j}=0$ for all $i \neq j$. Hence, $V_{N}^{*}(0) \leq V_{N}(0, \mathbf{L}, 0,0)=0$. Since $V_{N}^{*}(x) \geq 0 \forall x \in X_{N}^{s f}, V_{N}^{*}(0)=0$, thus $\mu_{N}(0)=0$.

\subsection{Input-to-State Stability (ISS) for $\mathrm{RHC}$}

Since the disturbance is non-zero, it is not possible to guarantee that the origin is asymptotically stable, as in conventional RHC without disturbances [24]. As an alternative, we use the notion of input-to-state stability (ISS) $[13,15]$, which has proven to be effective in the study of RHC laws with input constraints only [16] and in the analysis and synthesis of RHC laws with robust constraint satisfaction guarantees [23].

Consider a nonlinear, time-invariant, discrete-time system of the form

$$
x^{+}=f(x, w),
$$

where $x \in \mathbb{R}^{n}$ is the state and $w \in \mathbb{R}^{l}$ is a disturbance that takes on values in a compact set $W \subset \mathbb{R}^{l}$ containing the origin. It is assumed that the state is measured at each time instant, that $f: \mathbb{R}^{n} \times \mathbb{R}^{l} \rightarrow \mathbb{R}^{n}$ is continuous and that $f(0,0)=0$. Given the state $x$ at time 0 , a disturbance sequence $w(\cdot)$, where $w(k) \in W$ for all $k \in$
$\mathbb{Z}_{[0, \infty)}$, let the solution to (29) at time $k$ be denoted by $\phi(k, x, w(\cdot))$. For systems of this type, a useful definition of stability is input-to-state stability:

Definition 19 (ISS) For system (29), the origin is input-to-state stable (ISS) with region of attraction $\mathcal{X} \subseteq \mathbb{R}^{n}$, which contains the origin in its interior, if there exist a $\mathcal{K} \mathcal{L}$-function $\beta(\cdot)$ and a $\mathcal{K}$-function $\gamma(\cdot)$ such that for all initial states $x \in \mathcal{X}$ and disturbance sequences $w(\cdot)$, where $w(k) \in W$ for all $k \in \mathbb{Z}_{[0, \infty)}$, the solution of the system satisfies $\phi(k, x, w(\cdot)) \in \mathcal{X}$ and for all $k \in \mathbb{N}$,

$$
\begin{aligned}
& \|\phi(k, x, w(\cdot))\| \leq \beta(\|x\|, k)+ \\
& \quad \gamma\left(\sup \left\{\|w(\tau)\| \mid \tau \in \mathbb{Z}_{[0, k-1]}\right\}\right)
\end{aligned}
$$

Note that input-to-state stability implies that the origin is an asymptotically stable point for the undisturbed system $x^{+}=f(x, 0)$ with region of attraction $\mathcal{X}$, and also that all state trajectories are bounded for all bounded disturbance sequences. Furthermore, every trajectory $\phi(x, k, w(\cdot)) \rightarrow 0$ if $w(k) \rightarrow 0$ as $k \rightarrow \infty$.

In order to be self-contained, we recall the following useful result from [13, Lem 3.5]:

Lemma 20 (ISS-Lyapunov function) For the system (29), the origin is ISS with region of attraction $\mathcal{X} \subseteq \mathbb{R}^{n}$ if the following conditions are satisfied:

- $\mathcal{X}$ contains the origin in its interior and $X_{f}$ is robust positively invariant for (29), i.e. $f(x, w) \in \mathcal{X}$ for all $x \in \mathcal{X}$ and all $w \in W$.

- There exist $\mathcal{K}_{\infty}$ functions $\alpha_{1}(\cdot), \alpha_{2}(\cdot)$ and $\alpha_{3}(\cdot)$, a $\mathcal{K}$ function $\sigma(\cdot)$, and a continuous function $V: \mathcal{X} \rightarrow \mathbb{R}_{\geq 0}$ such that for all $x \in \mathcal{X}$,

$$
\begin{aligned}
\alpha_{1}(\|x\|) & \leq V(x) \leq \alpha_{2}(\|x\|) \\
V(f(x, w)) & -V(x) \leq-\alpha_{3}(\|x\|)+\sigma(\|w\|)
\end{aligned}
$$

Remark 21 A function $V(\cdot)$ that satisfies the conditions in Lemma 20 is called an ISS-Lyapunov function.

The above result leads immediately to the following:

Lemma 22 [Lipschitz Lyapunov function for undisturbed system] Let $\mathcal{X} \subseteq \mathbb{R}^{n}$ contain the origin in its interior and be a robust positively invariant set for (29). Furthermore, let there exist $\mathcal{K}_{\infty}$-functions $\alpha_{1}(\cdot), \alpha_{2}(\cdot)$ and $\alpha_{3}(\cdot)$ and a function $V: \mathcal{X} \rightarrow \mathbb{R}_{\geq 0}$ that is Lipschitz continuous on $\mathcal{X}$ such that for all $x \in \mathcal{X}$,

$$
\begin{gathered}
\alpha_{1}(\|x\|) \leq V(x) \leq \alpha_{2}(\|x\|) \\
V(f(x, 0))-V(x) \leq-\alpha_{3}(\|x\|)
\end{gathered}
$$

The function $V(\cdot)$ is an ISS-Lyapunov function and the origin is ISS for the system (29) with region of attraction $\mathcal{X}$ if either of the following conditions are satisfied: 
(i) $f: \mathcal{X} \times W \rightarrow \mathbb{R}^{n}$ is Lipschitz continuous on $\mathcal{X} \times W$.

(ii) $f(x, w):=g(x)+w$, where $g: \mathcal{X} \rightarrow \mathbb{R}^{n}$ is continuous on $\mathcal{X}$.

PROOF. Let $L_{V}$ be the Lipschitz constant of $V(\cdot)$. (i) Since $\|V(f(x, w))-V(f(x, 0))\| \leq L_{V} \| f(x, w)-$ $f(x, 0)\left\|\leq L_{V} L_{f}\right\| w \|$, where $L_{f}$ is the Lipschitz constant of $f(\cdot)$, it follows that $V(f(x, w))-V(x)=V(f(x, 0))-$ $V(x)+V(f(x, w))-V(f(x, 0)) \leq-\alpha_{3}(\|x\|)+L_{V} L_{f}\|w\|$. The proof is completed by letting $\sigma(s):=L_{V} L_{f} s$ in Lemma 20.

(ii) Note that $\|V(f(x, w))-V(f(x, 0))\| \leq L_{V}\|w\|$. The proof is completed as for (i), but by letting $\sigma(s):=L_{V} s$ in Lemma 20.

Finally, we add the following assumption, which allows $V_{N}^{*}(\cdot)$ in $(25)$ to be used as an ISS-Lyapunov function:

A2 (Terminal cost) The terminal cost $F(x):=x^{T} P x$ is a Lyapunov function in the terminal set $X_{f}$ for the undisturbed closed loop system $x^{+}=(A+B K) x$ in the sense that, for all $x \in X_{f}$,

$$
F((A+B K) x)-F(x) \leq-x^{T}\left(Q+K^{T} R K\right) x
$$

Theorem 23 (ISS for RHC) Let $W$ be a polytope and the RHC law $\mu_{N}(\cdot)$ be defined as in (23). If $\boldsymbol{A} \mathbf{1}$ and $\boldsymbol{A} 2$ hold, then the origin is ISS for the closed-loop system (24) with region of attraction $X_{N}^{s f}$. Furthermore, the input and state constraints (2) are satisfied for all time and for all allowable disturbance sequences if and only if the initial state $x(0) \in X_{N}^{s f}$.

PROOF. For the system of interest, we of course let $f(x, w):=A x+B \mu_{N}(x)+w$. Lemma 18 implies that $f(0,0)=0$ and Proposition 17 implies that $f(\cdot)$ is continuous on $X_{N}^{s f}$.

Combining Proposition 17 with Lemma 18, it follows that $V_{N}^{*}(\cdot)$ is a continuous, positive definite function. Hence, there exist $\mathcal{K}_{\infty}$-functions $\alpha_{1}(\cdot)$ and $\alpha_{2}(\cdot)$ such that (30a) holds with $V(\cdot):=V_{N}^{*}(\cdot)[15$, Lem. 4.3].

Using standard techniques [24], it is easy to show that $V(\cdot):=V_{N}^{*}(\cdot)$ is a Lyapunov function for the undisturbed system $x^{+}=A x+B \mu_{N}(x)$. More precisely, the methods in [24] can be employed to show that (30b) holds with $\alpha_{3}(z):=(1 / 2) \lambda_{\min }(Q) z^{2}$.

It follows from Proposition 13 that $X_{N}^{s f}$ is robust positively invariant for system (24). Proposition 11 implies that the origin is in the interior of $X_{N}^{s f}$. Finally, recall from Proposition 17 that $\mu_{N}(\cdot)$ and $V_{N}^{*}(\cdot)$ are Lipschitz continuous on $X_{N}^{s f}$. By combining all of the above, it follows from Lemma 22 that $V_{N}^{*}(\cdot)$ is an ISS-Lyapunov function for system (24).

Remark 24 Given the same assumptions as in Theorem 23, it can be shown [24] that the origin is an exponentially stable equilibrium for the undisturbed system $x^{+}=A x+B \mu_{N}(x)$ with region of attraction $X_{N}^{s f}$.

\section{Conclusions}

We have shown that the state feedback parameterization of Section 3 is equivalent to the disturbance feedback parameterization of Section 4 . This has the important consequence that, under suitable assumptions on the disturbance and cost function in a given finite horizon optimal control problem, an admissible and optimal state feedback control policy can be found by solving a tractable and convex optimization problem. This is a surprising result, since the set of admissible affine state feedback parameters is non-convex, in general.

In addition, if the optimal control problem involves the minimization of a quadratic cost and the solution is to be implemented in a receding horizon fashion, then one can choose the terminal cost and terminal constraint to guarantee that the closed-loop system is input-tostate stable and that the state and input constraints are satisfied for all time and for all disturbance sequences.

A number of open research issues remain. For example, we have shown that the proposed disturbance feedback parameterization is equivalent to affine state-feedback with knowledge of prior states. It would be interesting to see if it is possible to derive an equivalent convex reparameterization in the case where the control at each stage is an affine function of the current state only.

This paper only considered the regulation problem with state feedback. In order to be practically useful, the results need to be extended to handle the cases of output feedback, setpoint tracking and offset-free control.

It may be possible to extend the continuity and stability results in Proposition 17 and Theorem 23 to cover a broader class of disturbances, such as ellipsoidal or 2norm bounded disturbances, or to cover more general convex constraints on the states and inputs. However, the arguments used for doing so are likely to differ substantially from those given here.

The result on computational tractability may be extended to exploit any additional structure in the optimal control problem for specific classes of disturbances and cost functions. Some results along these lines are already available for problems with $\infty$-norm bounded disturbances [10] and the minimization of the finite-horizon $\ell_{2}$ gain of a system [14]. 


\section{Acknowledgements}

Paul Goulart would like to thank the Gates Cambridge Trust for their support. Eric Kerrigan would like to thank the Royal Academy of Engineering for supporting this research.

\section{References}

[1] A. Bemporad. Reducing conservativeness in predictive control of constrained systems with disturbances. In Proc. 37th IEEE Conf. on Decision and Control, pages 1384-1391, Tampa, FL, USA, December 1998.

[2] A. Bemporad, M. Morari, V. Dua, and E.N. Pistikopoulos. The explicit linear quadratic regulator for constrained systems. Automatica, 38(1):3-20, January 2002.

[3] A. Ben-Tal, A. Goryashko, E. Guslitzer, and A Nemirovski. Adjustable robust solutions of uncertain linear programs. Mathematical Programming, 99(2):351-376, March 2004.

[4] D. P. Bertsekas and I. B. Rhodes. Sufficiently informative functions and the minimax feedback control of uncertain dynamic systems. IEEE Transactions on Automatic Control, AC-18(2):117-124, April 1973.

[5] F. Blanchini. Set invariance in control. Automatica, 35(1):1747-1767, November 1999.

[6] L. Chisci, J. A. Rossiter, and G. Zappa. Systems with persistent state disturbances: predictive control with restricted constraints. Automatica, 37(7):1019-1028, July 2001.

[7] M.A. Dahleh and I.J. Diaz-Bobillo. Control of Uncertain Systems. Prentice Hall, 1995.

[8] Bertsekas D.P., Nedic A., and Ozdaglar A.E. Convex Analysis and Optimization. Athena Scientific, 2003.

[9] S. J. Gartska and R. J-B. Wets. On decisions rules in stochastic programming. Mathematical Programming, 7:117143, 1974.

[10] P. J. Goulart and E. C. Kerrigan. An efficient decompositionbased formulation for robust control with constraints. In Proc. 16th IFAC World Congress on Automatic Control, Prague, Czech Repulic, July 2005.

[11] E. Guslitser. Uncertainty-immunized solutions in linear programming. Master's thesis, Technion, Israeli Institute of Technology, June 2002.

[12] D. Jia, B. H. Krogh, and O. Stursberg. An LMI approach to robust model predictive control. Journal of Optimization Theory and Applications, 2005. In press.

[13] Z. Jiang and Y. Wang. Input-to-state stability for discretetime non-linear systems. Automatica, 37(6):857-869, June 2001.

[14] E. C. Kerrigan and T. Alamo. A convex parameterization for solving constrained min-max problems with a quadratic cost. In Proc. 2004 American Control Conference, Boston, MA, USA, June 2004.

[15] H.K. Khalil. Nonlinear Systems. Prentice Hall, USA, 2002.

[16] J. Kim, T. Yoon, A. Jadbabaie, and C. De Persis. Inputto-state stabilizing MPC for neturally stable linear systems subject to input constraints. In Proc. 43rd IEEE Conference on Decision and Control, pages 5041-5046, Paradise Island, Bahamas, December 2004.
[17] I. Kolmanovsky and E. G. Gilbert. Theory and computations of disturbance invariant sets for discrete-time linear systems. Mathematical Problems in Engineering, 4(4):317-363, 1998.

[18] M. V. Kothare, V. Balakrishnan, and M. Morari. Robust constrained model predictive control using linear matrix inequalities. Automatica, 32(10):1361-1379, October 1996.

[19] Y. I. Lee and B. Kouvaritakis. Constrained receding horizon predictive control for systems with disturbances. International Journal of Control, 72(11):1027-1032, August 1999.

[20] M. S. Lobo, L. Vandenberghe, S. Boyd, and H. Lebret. Applications of second-order cone programming. Linear Algebra and its Applications, 284(1-3):193-228, November 1998.

[21] J. Löfberg. Approximations of closed-loop MPC. In Proc. 42nd IEEE Conference on Decision and Control, pages 14381442, Maui, Hawaii, USA, December 2003.

[22] J. M. Maciejowski. Predictive Control with Constraints. Prentice Hall, UK, 2002.

[23] D. Limón Marruedo, T. Álamo, and E. F. Camacho. Inputto-state stable MPC for constrained discrete-time nonlinear systems with bounded additive disturbances. In Proc. 41st IEEE Conference on Decision and Control, pages 4619-2624, Las Vegas, Nevada USA, December 2002.

[24] D. Q. Mayne, J. B. Rawlings, C. V. Rao, and P. O. M. Scokaert. Constrained model predictive control: Stability and optimality. Automatica, 36(6):789-814, June 2000. Survey paper.

[25] D. Q. Mayne, M. M. Seron, and S. V. Raković. Robust model predictive control of constrained linear systems with bounded disturbances. Automatica, 41(2):219-24, February 2005.

[26] P. O. M. Scokaert and D. Q. Mayne. Min-max feedback model predictive control for constrained linear systems. IEEE Transactions on Automatic Control, 43(8):1136-1142, August 1998.

[27] R. S. Smith. Robust model predictive control of constrained linear systems. In Proc. 2004 American Control Conference, Boston, MA, USA, June 2004.

[28] D. H. van Hessem and O. H. Bosgra. A conic reformulation of model predictive control including bounded and stochastic disturbances under state and input constraints. In Proc. $41 s t$ IEEE Conference on Decision and Control, pages 4643-4648, December 2002.

\section{Appendix}

Define $\mathbf{A} \in \mathbb{R}^{n(N+1) \times n}$ and $\mathbf{E} \in \mathbb{R}^{n(N+1) \times n N}$ as

$$
\mathbf{A}:=\left[\begin{array}{c}
I_{n} \\
A \\
A^{2} \\
\vdots \\
A^{N}
\end{array}\right], \quad \mathbf{E}:=\left[\begin{array}{cccc}
0 & 0 & \cdots & 0 \\
I_{n} & 0 & \cdots & 0 \\
A & I_{n} & \cdots & 0 \\
\vdots & \vdots & \ddots & \vdots \\
A^{N-1} & A^{N-2} & \cdots & I_{n}
\end{array}\right]
$$

The matrices $\mathbf{B} \in \mathbb{R}^{n(N+1) \times m N}, \mathbf{C} \in \mathbb{R}^{t \times n(N+1)}$ and $\mathbf{D} \in \mathbb{R}^{t \times m N}$ are defined as $\mathbf{B}:=\mathbf{E}\left(I_{N} \otimes B\right)$, $\mathbf{C}:=\left[\begin{array}{cc}I_{N} \otimes C & 0 \\ 0 & Y\end{array}\right]$, and $\mathbf{D}:=\left[\begin{array}{c}I_{N} \otimes D \\ 0\end{array}\right]$. It is easy to verify that (11) is equivalent to (13) with $F:=\mathbf{C B}+\mathbf{D}$, $G:=\mathbf{C E}, H:=-\mathbf{C A}$, and $c:=\left[\begin{array}{c}\mathbf{1}_{\mathrm{N}} \otimes b \\ z\end{array}\right]$. 